\title{
EBM II: How to perform a literature search
}

M. Castagnetti, K.W. Herbst, D. Bagli, G.M.A. Beckers, L. Harper, M. Kaefer, N. Kalfa and M. Fossum

\section{Summary}

The present article is a second part related to evidence based medicine (EBM) in a series of five by the European society for paediatric urology (ESPU) research committee. It will present the different databases/search engines available to clinicians and researchers and describe strategies to focus the search to one's particular needs. Indeed, databases/search engines used and search strategy should vary according to the goal of the research. If the aim is to address a clinical problem, the search should allow to identify a small number of most pertinent articles (high specificity); if the search is for research purposes, instead, it should ensure no meaningful articles are overlooked (high sensitivity).

Following a previous article, the present article will focus here on literature search ${ }^{1}$.

Ideally, a literature research should find a balance between being broad enough to identify all relevant information (sensitivity) yet not yield so many articles to make scrutiny of results impossible (specificity). Sensitivity and specificity should be modulated to the aim of the search. If the aim is to address a clinical problem, greater specificity is needed to identify only the most pertinent articles; if the search is for a systematic review, high sensitivity is required to ensure no meaningful articles are overlooked. 
To balance these opposite goals, it is important to know available search tools and databases and how to narrow a search. This means knowing where to search and how to search.

Where to search: PubMed/Medline is the most commonly used database of medical literature, but is not the only source or always the most appropriate. For instance, if a search is to address a clinical question, searching clinical guideline databases such as EBM Guidelines, or ones that summarize evidence, such as the BMJ Clinical Evidence or BestBETs, might be more useful. Databases on specific topics, including CANCERLIT (cancer literature), CINAHL (nurses literature), or PsycINFO (literature on psychology), may identify articles not included in PubMed/Medline. The Cochrane Library contains systematic reviews performed under rigorous method protocols. The Cochrane Library also contains other databases such as the Cochrane Central Register of Controlled Trials (CENTRAL) that can be useful before embarking on a project to determine if a trial on the same topic is currently ongoing.

Search engines other than PubMed/Medline, such as Embase (Elsevier), Current Contents (Ovid), Scopus, Web of Science, Google Scholar, and Trip Pro, can also be important as different search engines may yield different results with the same key words. This is because some engines, such as Google Scholar, OVID, and Trip Pro, search across multiple databases or search for practice guidelines, books, medical image databases, dissertations, patient information, etc. in addition to journal articles, and not all journals are represented in PubMed. Searching multiple engines/databases is mandatory if the search is performed to make a systematic review.

How to search: Searching whole questions often yields unusable results as the query is based on a combination of multiple words. Therefore, one should strive to identify key words that 
pinpoint, in the most unambiguous way, the subject of your search. PubMed's Medical Subject Headings (MeSH) are particularly useful. These are classification terms, so once the appropriate MeSH is identified, the search will be more specific and can be used with other search engines. The use of Boolean operators such as AND, OR, and NOT can be used to further focus a search. Limitations can be applied, such as article type (review, original research, etc), time period, gender, age range, language, etc. Finally, multiple searches can be combined to exclude repetitive items or select only items present in several searches. For more information, tutorials on search strategies are available $e^{2-5}$.

\section{Author statements}

\section{Ethical approval}

None sought.

\section{Funding}

None declared.

\section{Competing interests}

The authors present no conflict of interest. 


\section{References}

[1]. Fossum M., Herbst K., Harper L., Castagnetti M., Beckers G., Bagli D., et al: Evidencebased medicine I background. J Pediatr Urol 2019; 15: pp. 78-79

[2]. Webinar: PubMed for Clinicians, https://www.youtube.com/watch?v=HYW2 eUsK9E [accessed 14 January 2019].

[3]. Use MeSH to build a better PubMed query, https://www.youtube.com/watch?v=uyF8uQY9wys [accessed 14 January 2019].

[4]. PubMed advanced search builder, https://www.youtube.com/watch? $\mathrm{v}=\mathrm{dncRQ} 1 \mathrm{cobdc}$ [accessed 14 January 2019].

[5]. PubMed the filters sidebar, https://www.youtube.com/watch?v=696R9GbOyvA [accessed 14 January 2019]. 